\title{
Irradiation detection of herbal ingredients used in plant food supplements by Electron Spin Resonance on samples pre-treated with alcoholic extraction.
}

\author{
E. Bortolin ${ }^{\mathrm{a}, *}$, C. Cardamone ${ }^{\mathrm{b}}$, A.E. Chiaravalle ${ }^{\mathrm{c}}$, G. Deiana ${ }^{\mathrm{d}}$, M.T. Di Schiavi ${ }^{\mathrm{e}}$, M.C. D'Oca ${ }^{\mathrm{f}}$, \\ G. Marchesani ${ }^{\mathrm{c}}$, M.C. Quattrini ${ }^{\mathrm{a}}$, E. Sangiorgi ${ }^{\mathrm{g}}$, M. Tomaiuolo ${ }^{\mathrm{c}}$, C. Boniglia ${ }^{\mathrm{a}}$

 \\ ${ }^{\mathrm{b}}$ Istituto Zooprofilattico Sperimentale della Sicilia, Palermo, Italy \\ ${ }^{\mathrm{c}}$ Istituto Zooprofilattico Sperimentale della Puglia e della Basilicata, Foggia, Italy \\ ${ }^{\mathrm{d}}$ Istituto Zooprofilattico Sperimentale della Sardegna, Sassari, Italy \\ ${ }^{\mathrm{e}}$ Istituto Zooprofilattico Sperimentale del Lazio e della Toscana, Roma, Italy \\ ${ }^{\mathrm{f}}$ Università degli Studi di Palermo, Palermo, Italy \\ ${ }^{\mathrm{g}}$ Istituto Zooprofilattico Sperimentale della Lombardia e dell'Emilia Romagna, Brescia, Italy
}

\section{A R T I C L E I N F O}

\section{Keywords:}

Irradiated food

Plant food supplements

PFS

Electron Spin Resonance

ESR

\begin{abstract}
A B S T R A C T
This study aimed to verify the applicability of the EN 1787 method for the detection of irradiation in herbal ingredients used in Plant Food Supplements (PFSs). In matrices such as herbs and spices the main limit of the method is the presence of intrinsic radicals responsible for spurious signals leading to complex ESR spectra. To overcome this limit, before ESR measurement a treatment with alcohol has been proposed (Delincée and Soika, 2002; Ahn et al., 2012, 2014). As reported in the literature, this treatment is expected to reduce/eliminate the confounding signals so that the samples may be correctly classified. In this study the efficacy of the pre-treatment was tested on raw herbal ingredients largely used for PFSs, namely Camellia sinensis, Cinnamomum verum, Curcuma longa, Ginkgo biloba, Silybum marianum, Vaccinium myrtillus and Zingiber officinale. Non-irradiated and irradiated (5, $10 \mathrm{kGy}$ ) samples were analysed before and after pre-treatment. The results showed a general decrement of signal intensity. In some cases, this was associated with the elimination of some spurious signals, which, however, did not always ensue in an easier interpretation of the ESR spectra. Only for two matrices (Camellia sinensis and Vaccinium myrtillus) was alcoholic extraction crucial for the correct classification of the samples.
\end{abstract}

\section{Introduction}

Reliable methods for the detection of irradiated herbal ingredients used in plant food supplements (PFSs) are being required since, upon official checks set out by the European Directives 1999/2/EC (Directive, 1999a) and 1999/3/EC (Directive, 1999b), non-negligible percentages of irradiated PFSs and their ingredients have been found to be irradiated and incorrectly labelled (http://ec.europa.eu/food/ safety/biosafety/irradiation/reports.en; Boniglia et al., 2009). The EN Standard 1787 (EN 1787, 2000) is one of the analytical methods standardised by the European Committee for Standardization (CEN) to analyse vegetable matrices containing cellulose, and suitable -in principle- for the identification of said irradiated ingredients. EN 1787 uses the Electron Spin Resonance (ESR) technique, which reveals species containing unpaired electrons, such as radicals, through the absorption of electromagnetic energy from microwaves in the presence of a static magnetic field. In particular, this technique identifies the radical induced by ionizing radiation in cellulose; this radical yields an ESR spectrum characterised by a triplet with two weak (satellite) lines at a specific distance (about $6.0 \mathrm{mT}$ ) symmetrically located on the sides of an intense peak detectable in the non-irradiated samples as well. The method was validated through international trials for pistachio nut shells, strawberries and paprika (Raffi, 1992; Raffi et al., 1992; Schreiber et al., 1993; Linke et al., 1995; Linke et al., 1996; Schreiber et al., 1996), but it would be applicable, in principle, to all vegetables containing cellulose. In practice, however, the reliability of the EN 1787 standard in detecting irradiation is limited by a low cellulose content and/or the presence of moisture that favors radical recombination

\footnotetext{
* Corresponding author. Core Facilities, Istituto Superiore di Sanità, Rome, Italy.

E-mail address: emanuela.bortolin@iss.it (E. Bortolin).
} 
(Bortolin et al., 2006). In addition, in some matrices, e.g., certain herbs and spices, the presence of intrinsic radicals induces spurious ESR signals overlapping those indicated in the EN 1787 standard, which makes it difficult to analyse spectra and classify samples (Delincée and Soika, 2002; Polovka et al., 2007; Yordanov et al., (2009); Ahn et al., 2012; Sanyal et al., 2012; Ahn et al., 2014; Kim et al., 2014; Sanyal et al., 2014). To overcome this problem, sample pre-treatment with alcohol before ESR measurement has been recently tested on some of herbal, spice and fruit matrices. The treatment was firstly proposed by de Jesus et al. (1999) to extend the applicability of EN 1787 to kiwi, papaya and tomato by using fruit pulp. It was then tested -also in combination with other treatments (water washing before alcoholic extraction)- on other matrices such as strawberries (Delincée and Soika, 2002), oranges (Jo et al., 2016), pomegranates (Shahbaz et al., 2013), sauces (Akram et al., 2013), herbs and spices (Delincée and Soika, 2002; Ahn et al., 2012, 2014). The results were not always satisfactory. Regarding herbs and spices, in particular, the effect observed seemed to depend on the matrix, which is not surprising given the large variety of components (including radicals) in these products. In fact, in an early work Delincée and Soika (2002) tested the procedure on a number of herbs and spices, namely chives, parsley, thyme, ground cinnamon, ground cumin, granulated garlic, ground nutmeg, whole and ground black pepper and ground white pepper, but only in a few cases (parsley and granulated garlic) was an improvement observed in the ESR spectra. Later on, Ahn et al. (2012) found a little improvement for red and white ginseng after alcoholic extraction, whereas in a successive work (Ahn et al., 2014) they reported very good results for turmeric, oregano and cinnamon pre-treated in the same way.

Taking into account the above results from the literature, the present study aimed to verify the efficacy of the pre-treatment with alcohol in improving the reliability of the EN 1787 method for the detection of irradiation in PFS ingredients. The validation of the method for these matrices would be crucial, as this analytical procedure is simple and fast, even with pre-treatment with alcohol, and once validated does not require confirmation of the results. It would therefore constitute a valid alternative to EN 13751 (EN 13751, 2009) and EN 1788 (EN 1788, 2001) that use stimulated luminescence. The EN 13751 method uses Photo-Stimulated Luminescence (PSL) and gives correct classifications for raw materials (part of plants, leaves, seeds, fruits, radix, etc.), but fails to do so with herbal extracts for they are poor in mineral contaminants (Sanderson et al., 2003a; Bortolin et al., 2009; Boniglia et al., 2018). Moreover, it requires a confirmation of non-negative results through a second measurement after laboratory irradiation (Calibrated PSL) or the re-analysis of the samples by another confirmatory method. Conversely, the EN 1788 method, which uses Thermoluminescence (TL) and is extremely reliable also for herbal extracts (Schreiber et al., 1996; Sanderson et al., 2003b), is time-consuming: it requires silicate extraction from foodstuffs to avoid the spurious signals due to the combustion of the organic part, and a second calibration measurement.

The present study focused on specific herbal ingredients chosen among the most commonly used in PFSs: Camellia sinensis, Cinnamomum verum, Curcuma longa, Ginkgo biloba, Silybum marianum, Vaccinium myrtillus, Zingiber officinale. To verify the efficacy of the alcoholic extraction, non-irradiated and irradiated (5, $10 \mathrm{kGy})$ samples were analysed before and after pre-treatment. The work was done in two steps: after a preliminary intra-laboratory validation of the analytical procedure, four Italian laboratories involved in official controls participated in an inter-laboratory blind test. A total of 48 samples -16 non-irradiated samples, 16 samples irradiated at $5 \mathrm{kGy}$ and 16 samples irradiated at $10 \mathrm{kGy}$ - were analysed by the four laboratories before and after alcoholic extraction.

\section{Materials and methods}

\subsection{Samples}

The samples of Camellia sinensis (leaves), Ginkgo biloba (leaves), Silybum marianum (fruits), Vaccinium myrtillus (fruits), Cinnamomum verum (powder), Zingiber officinale (radix), Curcuma longa (powder) were purchased from herbalists in Rome. All the products were stored at room conditions inside their original packaging.

\subsection{Irradiation}

The samples were irradiated at room conditions with doses of 5 and 10 kGy using a low-energy X-ray irradiator (RS 2150 Rad source Inc.) operating at $150 \mathrm{kV}$ and $45 \mathrm{~mA}$. The dose rate was $15 \mathrm{~Gy}$ min- 1 measured with a calibrated ionisation chamber (Rad-cal Inc.). For the irradiation the samples were placed as received in sealed plastic bags. Before being inserted into the plastic bags the products were crushed by hand if necessary. The uniformity of the dose was obtained by irradiating the food matrices inside a carousel rotating around the X-ray tube. Dosimetry evaluations were carried out with alanine pellets ( $5 \mathrm{~mm}$ diameter, Bruker, Rheinstetten, Germany). The absorbed doses reported in this work are doses to water with an uncertainty of about $5 \%$. After irradiation the products were stored in their sealed plastic bags at room conditions.

\subsection{Validation plan}

The analytical procedure including the alcoholic extraction was set up through intra-laboratory tests performed by two laboratories. To verify the efficacy of the alcoholic extraction, non-irradiated and irradiated $(5,10 \mathrm{kGy})$ samples were analysed before and after the treatment within two months from irradiation. Before the analysis the samples were stored in their sealed plastic bags at room conditions. The alcoholic extraction was performed following the procedure described in Ahn et al. (2014) and reported in section 2.4. ESR spectra were recorded under room conditions using the parameters indicated in the EN 1787 Standard and reported in section 2.5.

Successively, to validate the method, a blind inter-laboratory test was organised among four Italian laboratories involved in the official control of foods. Sixteen non-irradiated samples, 16 samples irradiated at $5 \mathrm{kGy}$ and 16 samples irradiated at $10 \mathrm{kGy}$ were sent to the participants who were asked to analyse each and every sample before and after the alcoholic extraction within two months from irradiation. A form for data collection was sent to each laboratory to report the results of the analyses and measurement conditions (sample mass, recording parameters, etc.).

\subsection{Alcoholic extraction procedure}

The alcoholic extraction was carried out as follows: about $3 \mathrm{~g}$ of sample were introduced in $20 \mathrm{ml}$ of an $80 \%$ alcohol solution, kept in solution for $30 \mathrm{~min}$ under magnetic stirring and subsequently filtered, pressed and dried in the oven at about $40{ }^{\circ} \mathrm{C}$ for an hour.

\subsection{ESR measurements}

ESR spectra were recorded with different models of Bruker spectrometers operating in the $\mathrm{X}$ band, depending on the equipment of the laboratory: Elexsys (one laboratory), E-Scan Food Analyzer (four laboratories) and ESR Bruker EMX (one laboratory). Measurements were done at room temperature and humidity, setting the parameters that follow as indicated in the EN 1787 Standard.

Frequency: about $9.8 \mathrm{GHz}$; center field: about $350 \mathrm{mT}$; sweep width: about $20 \mathrm{mT}$; microwave power: $0.4-0.8 \mathrm{~mW}$; modulation amplitude: 0.4-0.9 mT; sweep speed: $5 \mathrm{mT} / \mathrm{min}-10 \mathrm{mT} / \mathrm{min}$. 
Table 1

Intra-laboratory validation - Classification of non-irradiated samples.

\begin{tabular}{lll}
\hline Matrices & Before alcoholic extraction & After alcoholic extraction \\
\hline Camellia sinensis & non-irradiated & non-irradiated \\
Cinnamomum verum & indeterminate & indeterminate \\
Curcuma longa & indeterminate & non-irradiated \\
Ginkgo biloba & indeterminate & indeterminate \\
Silybum marianum & non-irradiated & non-irradiated \\
Vaccinium myrtillus & non-irradiated & non-irradiated \\
Zingiber officinale & non-irradiated & non-irradiated \\
\hline
\end{tabular}

Table 2

Intra-laboratory validation - Classification of 5 and $10 \mathrm{kGy}$ irradiated samples.

\begin{tabular}{lll}
\hline Matrices & Before alcoholic extraction & After alcoholic extraction \\
\hline Camellia sinensis & indeterminate & irradiated \\
Cinnamomum verum & irradiated & irradiated \\
Curcuma longa & indeterminate & indeterminate \\
Ginkgo biloba & indeterminate & indeterminate \\
Silybum marianum & irradiated & irradiated \\
Vaccinium myrtillus & indeterminate & irradiated \\
Zingiber officinale & irradiated & indeterminate \\
\end{tabular}

The samples were kept at about $40{ }^{\circ} \mathrm{C}$ for about $1 \mathrm{~h}$ to reduce the moisture content of the matrices. Then they were cut into small pieces, weighted and inserted in special tubes (glass or quartz) of 4-5 mm of diameter for measurement. Different aliquots of about $100 \mathrm{mg}$ (within $10 \%$ ) were used for each product for the analyses with and without alcoholic extraction.

\section{Results and discussion}

\subsection{Intra-laboratory validation}

The products analysed in this study showed ESR spectra generally complex and different, which reflect the complexity and variability of the chemical composition of these matrices. However, both laboratories involved in this preliminary study obtained, for each matrix, ESR spectra very similar by using different ESR spectrometers, so confirmed the reproducibility of the analyses.

Tables 1 and 2 and Figs. 1-7 show the results obtained, before and after alcoholic extraction, with both non-irradiated and irradiated matrices. As expected from the literature, the ESR spectra obtained before the alcoholic extraction generally appeared rather complex, with several overlapping signals, which made their analysis and sample classification difficult. As for non-irradiated samples, most of them (4 out of 7) showed spectra rather "clean" where only the central peak was visible. Consequently, these samples could be correctly classified. Three of the products analysed, however, i.e, Cinnamomum verum, Curcuma longa and Ginkgo biloba, showed spectra by which sample status (nonirradiated/irradiated) could not be established with certainty. As for the irradiated samples, only in 3 out of 7 were the radiation-induced ESR satellite lines clearly recognized before the treatment with alcohol. ESR spectra of the same herbal ingredient irradiated at different doses showed the same features; however, the radiation-induced signal was more visible at $10 \mathrm{kGy}$.

As an example, Fig. 1 shows the ESR spectra recorded before alcoholic extraction of non-irradiated and irradiated samples of Ginkgo biloba: the spectra show none of the features reported in the EN 1787 standard, and do not distinguish the non-irradiated samples from the irradiated ones.

After alcoholic extraction, the ESR spectra of both non-irradiated and irradiated samples showed an overall signal intensity reduction which, in some cases, led to the disappearance of some confounding signals. For the irradiated samples, this led, in some cases, to a better resolution of the radiation-induced triplet, which was more intense and easier to detect, regardless of the dose. The disappearance of some "spurious" signals, however, did not always guarantee the correct classification of the sample. Only in two cases (Camellia sinensis and Vaccinium myrtillus) was the alcoholic extraction crucial for the identification of the samples. Figs. 2 and 3 report the ESR spectra of irradiated Camellia sinensis and Vaccinium myrtillus where the alcoholic extraction made evident the left satellite line, which was not clearly detectable before the treatment.

Conversely, irradiated Curcuma longa, Ginkgo biloba (Fig. 4) and Zingiber officinale (Fig. 5) showed rather complex spectra after alcoholic treatment. The spectra of Curcuma longa and Zingiber officinale appeared even more complex after alcoholic extraction; in the spectrum of Zingiber officinale, in particular, after the alcoholic extraction the left satellite line, visible before the alcoholic treatment at a distance of about $6 \mathrm{mT}$ from the right one, was no longer clearly identifiable (Fig. 5). Irradiated Cinnamomum verum and Silybum marianum showed no significant variations in their ESR spectra after alcoholic extraction. For each product ESR spectra recorded at different doses showed no significant qualitative differences.

As for the non-irradiated samples, in most cases, "cleaner" spectra were obtained after alcoholic extraction. As an example, Fig. 6 reports the spectra of Vaccinium mirtyllus: the two spurious signals flanking the central peak, were no longer visible after alcoholic extraction.

Conversely, the spectra of Cinnamomum verum (Fig. 7) and Ginkgo biloba remained complex, even after the treatment with alcohol, and the status (non-irradiated/irradiated) of the samples could not be defined.

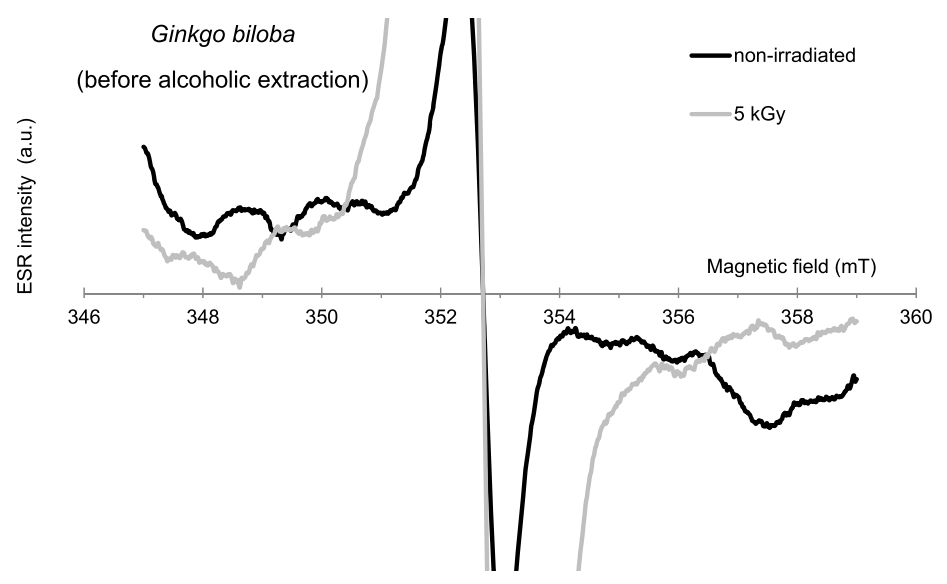

Fig. 1. ESR spectra of non-irradiated and irradiated Ginkgo biloba recorded before alcoholic extraction. 


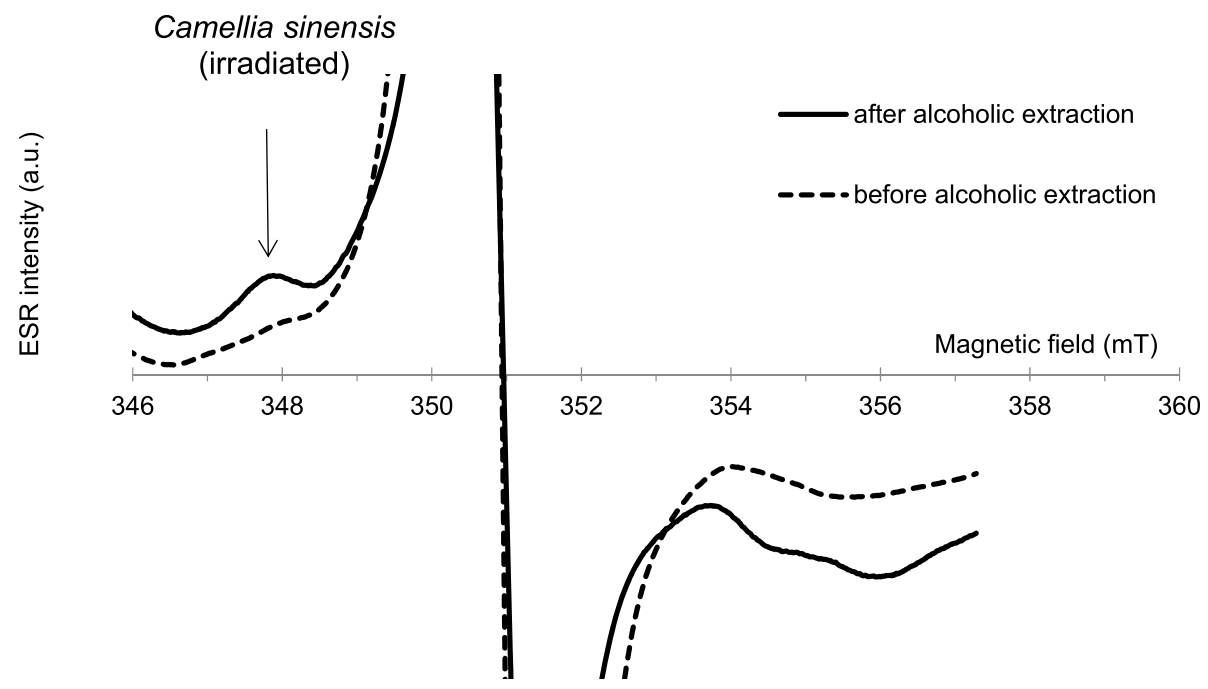

Fig. 2. ESR spectra of irradiated (10 kGy) Camellia sinensis recorded before and after alcoholic extraction.

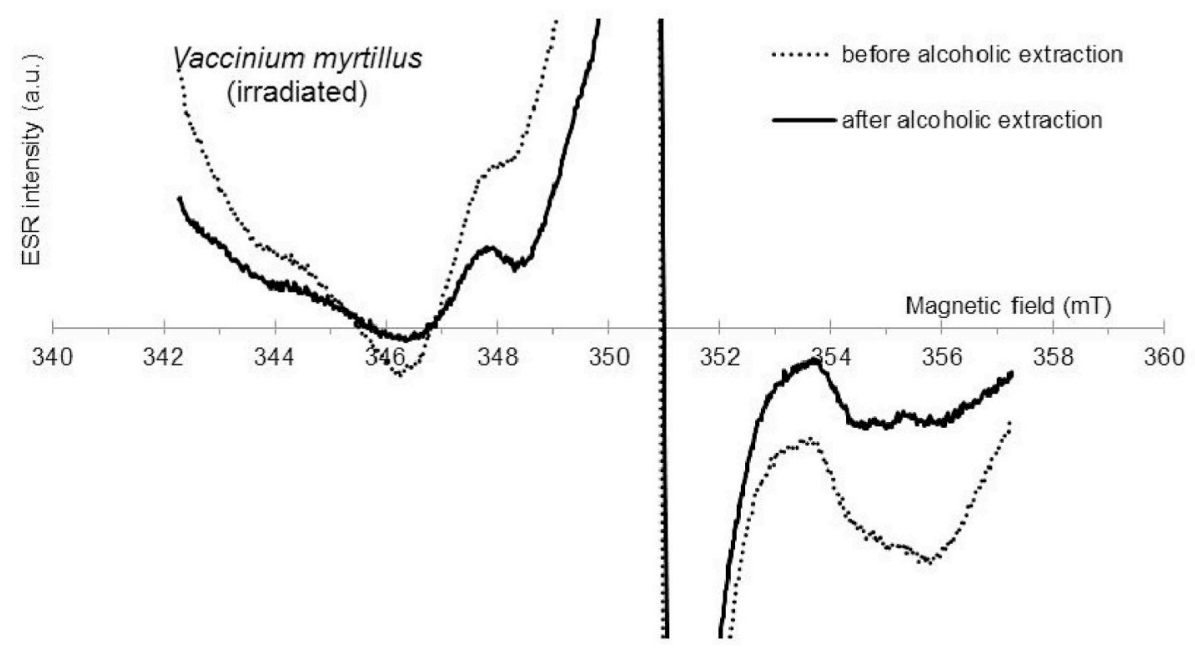

Fig. 3. ESR spectra of irradiated (10 kGy) Vaccinium myrtillus recorded before and after alcoholic extraction.



Fig. 4. ESR spectra of irradiated ( $5 \mathrm{kGy)} \mathrm{Ginkgo} \mathrm{biloba} \mathrm{recorded} \mathrm{before} \mathrm{and} \mathrm{after} \mathrm{alcoholic} \mathrm{extraction.}$ 


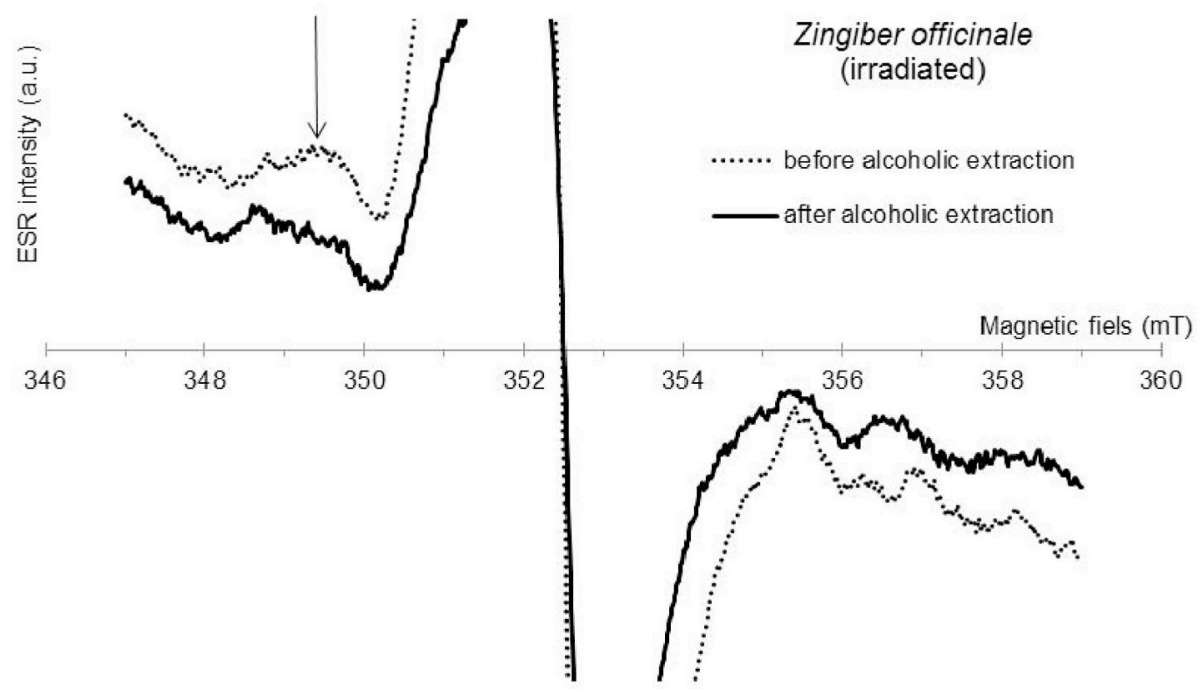

Fig. 5. ESR spectra of irradiated (10 kGy) Zingiber officinale recorded before and after alcoholic extraction.

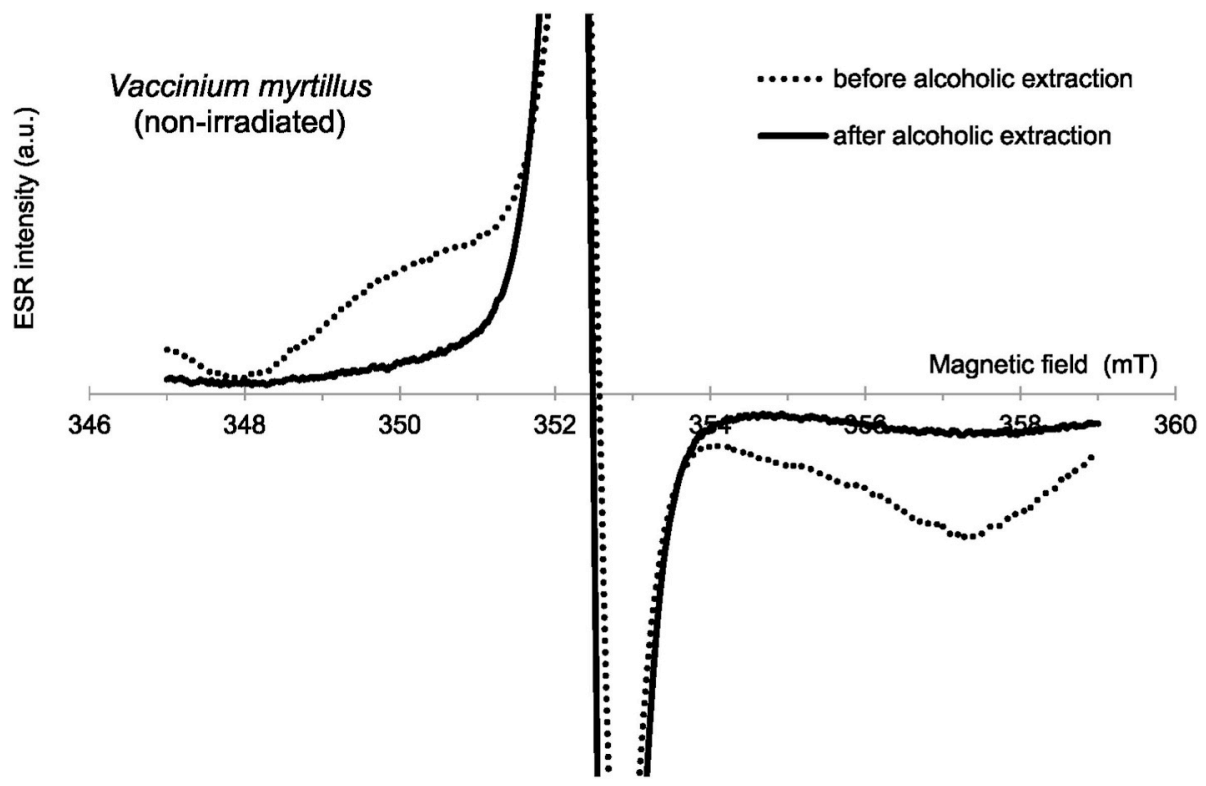

Fig. 6. ESR spectra of non-irradiated Vaccinium myrtillus recorded before and after alcoholic extraction.

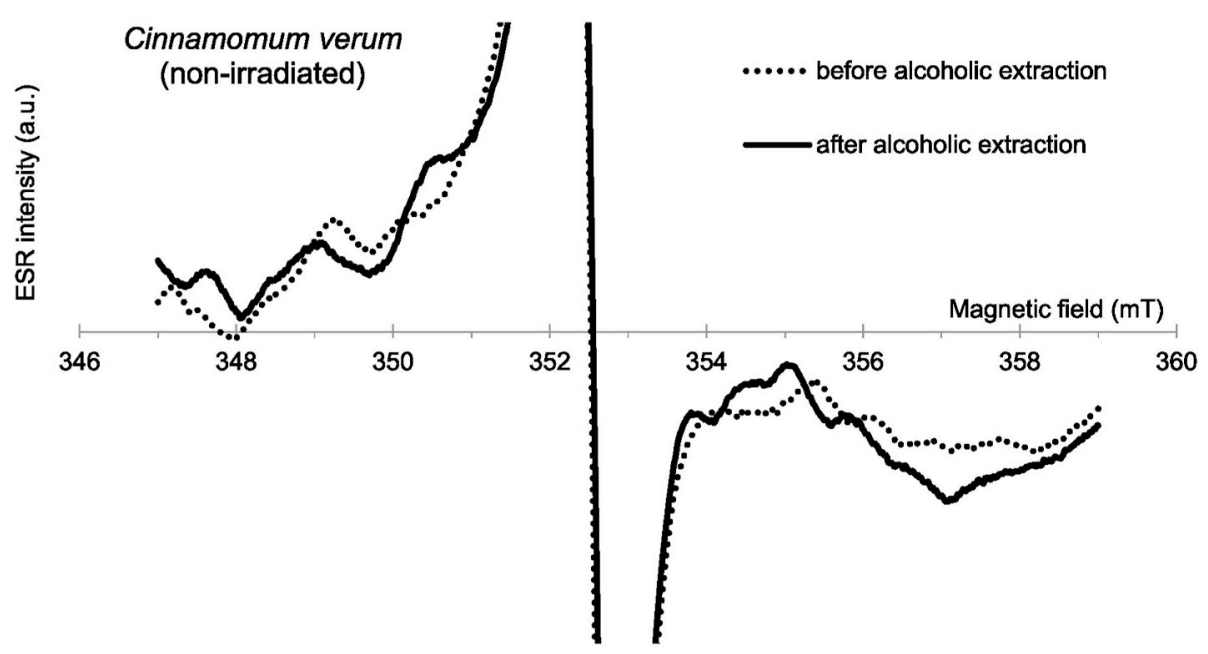

Fig. 7. ESR spectra of non-irradiated Cinnamomum verum recorded before and after alcoholic extraction. 
Table 3

Inter-laboratory validation - Correct classification (\%) of non-irradiated samples.

\begin{tabular}{lll}
\hline Matrices & Before alcoholic extraction & After alcoholic extraction \\
\hline Camellia sinensis & 100 & 100 \\
Ginkgo biloba & 75 & 50 \\
Silybum marianum & 100 & 100 \\
Vaccinium myrtillus & 100 & 100
\end{tabular}

Table 4

Inter-laboratory validation - Correct classification (\%) of irradiated samples.

\begin{tabular}{lll}
\hline Matrices & Before alcoholic extraction & After alcoholic extraction \\
\hline Camellia sinensis & 100 & 100 \\
Ginkgo biloba & 25 & 12.5 \\
Silybum marianum & 100 & 100 \\
Vaccinium myrtillus & 87.5 & 100 \\
\hline
\end{tabular}

\subsection{Inter-laboratory validation}

On the basis of the results obtained in the first part of the work, four matrices were selected for the inter-comparative test: Camellia sinensis and Vaccinium myrtillus on which the pre-treatment with alcohol was particularly effective, Silybum marianum that did not show significant variations in the spectrum, and Ginkgo biloba for which classifications remained indeterminate even after alcoholic extraction. For each matrix three samples were prepared: one non-irradiated, one irradiated at $5 \mathrm{kGy}$ and one irradiated at $10 \mathrm{kGy}$. Thus 48 samples in all -16 nonirradiated, 16 irradiated at $5 \mathrm{kGy}$ and 16 irradiated at $10 \mathrm{kGy}$ - were sent to the four laboratories involved in the inter-comparison exercise.

The results obtained by the participants (Tables 3 and 4) confirmed what had been observed in the first part of the work: in particular, an improvement after the treatment with alcohol in the irradiated samples of Camellia sinensis and Vaccinium myrtillus, whose ESR spectra appeared "cleaner" than before the extraction.

The treatment was particularly effective in irradiated Vaccinium myrtillus: the percentage of correct classifications increased from $87.5 \%$ to $100 \%$. For Ginkgo biloba the alcoholic extraction not only did not improve the results but actually made them worse, with a percentage of correct classifications of irradiated samples dropping to $12.5 \%$.

\section{Conclusions}

The objective of this work was to verify whether the EN 1787 standard, based on ESR technique, was applicable for the detection of irradiation in PFS ingredients, as an alternative to the stimulated luminescence-based methods EN 13751 and EN 1788. In particular, the work aimed to verify the efficacy of a pre-treatment with alcohol in reducing/eliminate the "spurious" signals in order to facilitate spectra analysis and increase the number of unambiguous classifications. To this purpose, non-irradiated and irradiated samples of seven herbal ingredients, namely Camellia sinensis, Cinnamomum verum, Curcuma longa, Ginkgo biloba, Silybum marianum, Vaccinium myrtillus, Zingiber officinale, in the form of leaves, seeds, fruits and radix, were analysed before and after alcoholic extraction.

The samples analysed before alcoholic extraction showed ESR spectra generally complex and different, which reflect the complexity and variability of the chemical composition of these matrices.

After alcoholic extraction, the ESR spectra of both non-irradiated and irradiated samples showed an overall signal intensity reduction which, in some cases, led to the disappearance of some confounding signals. The elimination of the "spurious" signals, however, did not always guarantee the correct classification of the samples. Only in two cases (namely, Camellia sinensis, and Vaccinium myrtillus) was alcoholic extraction decisive for the identification of the irradiated sample. In other cases the procedure either had no significant effect on the spectra or made them worse, leading to a reduction of the correct classifications (Ginkgo biloba) during the inter-comparative test.

In conclusion, given that the alcoholic extraction procedure may improve the quality of the results and allow a correct, unambiguous interpretation of the spectra, we feel that it is worth verifying the reliability of the method on each single matrix before applying it for official controls.

\section{CRediT authorship contribution statement}

E. Bortolin: Funding acquisition, Conceptualization, Methodology, Investigation, Writing - original draft, Writing - review \& editing. C. Cardamone: Investigation. A.E. Chiaravalle: Funding acquisition, Conceptualization, Methodology. G. Deiana: Investigation. M.T. Di Schiavi: Investigation. M.C. D'Oca: Investigation. G. Marchesani: Investigation. M.C. Quattrini: Investigation. E. Sangiorgi: Investigation. M. Tomaiuolo: Funding acquisition, Conceptualization, Methodology, Investigation, Writing - original draft, Writing - review \& editing. C. Boniglia: Funding acquisition, Conceptualization, Methodology, Investigation, Writing - original draft, Writing - review \& editing.

\section{Acknowledgments}

This research was partially funded by the Ministero della Salute (Italy), grant ID I85D18000200005.

The authors are grateful to Ms. Monica B. Brocco for the English editing of the manuscript.

\section{Appendix A. Supplementary data}

Supplementary data to this article can be found online at https:// doi.org/10.1016/j.radphyschem.2020.108946.

\section{References}

Ahn, J.J., Akram, K., Jo, D., Kwon, J.H., 2012. Investigation of different factors affecting the electron spin resonance-based characterization of gamma-irradiated fresh, white and red ginseng. J. Ginseng Res. 36 (3), 308-312.

Ahn, J.J., Sanyal, B., Akram, K., Kwon, J.H., 2014. Alcoholic extraction enables EPR analysis to characterize radiation induced cellulosic signals in spices. J. Agric. Food Chem. 62, 11089-11098.

Akram, K., Ahn, J.J., Kwon, J.H., 2013. Characterization and identification of gammairradiated sauces by electron spin resonance spectroscopy using different sample pretreatments. Food Chem. 138, 1878-1883.

Boniglia, C., Aureli, P., Bortolin, E., Onori, S., 2009. Verification of imported food upon import for radiation processing: dried herbs, including herbs used in food supplements, and spices by PSL and TL. Radiat. Phys. Chem. 78, 679-681.

Boniglia, C., Carratù, B., Gargiulo, R., Bortolin, E., 2018. Detection of Irradiated Herbal Ingredients of Plant Food Supplements by Thermoluminescence Technique. Food Supplements Containing Botanicals: Benefits, Side Effects and Regulatory Aspects. Patrizia Restani Editor. The Scientific Inheritance of the EU Project PlantLIBRA. Springer.

Bortolin, E., Bustos Griffin, E., Cruz-Zaragoza, E., De Coste, V., Onori, S., 2006. Electron paramagnetic resonance detection of Mexican irradiated spices. Int. J. Food Sci. Technol. 41, 375-382.

Bortolin, E., Boniglia, C., Gargiulo, R., Onori, S., 2009. Herbal materials used in dietary supplements: comparison of luminescence methods for detection of irradiation. Radiat. Phys. Chem. 78, 683-685.

de Jesus, E.F.O., Rossi, A.M., Lopes, R.C., 1999. An ESR identification of gamma-irradiated kiwi, papaya and tomato using fruit pulp. Int. J. Food Sci. Technol. 34, 173-178.

Delincée, H., Soika, C., 2002. Improvement of the ESR detection of irradiated food, containing cellulose employing a simple extraction method. Radiat. Phys. Chem. 63, 437-441.

Directive, 1999a. EC of the European Parliament and of the Council of 22 February 1999 on the approximation of the laws of the member states concerning foods and food ingredients treated with ionizing radiation (Directive, 1999a). Off. J. Eur. Communities - Legislation 66, 16-22.

Directive, 1999b. EC of the European Parliament and of the Council of 22 February 1999 on the establishment of a Community list of the laws of foods and food ingredients treated with ionizing radiation (Directive, 1999b). Off. J. Eur. Communities - 
Legislation 66, 24-25.

En 1787, 2000. Detection of Irradiated Food Containing Cellulose by ESR Spectroscopy. European Committee for Standardization Brussels, Belgium.

En 1788, 2001. Thermoluminescence Detection of Irradiated Food from Which Silicate Minerals Can Be Isolated. European Committee for Standardization Brussels, Belgium.

En 13751, 2009. Detection of Irradiated Food Using Photostimulated Luminescence. European Committee for Standardization Brussels, Belgium. http://ec.europa.eu/ food/safety/biosafety/irradiation/reports_en.

Jo, Y., Sanyal, B., Park, H.J., Kwon, J.H., 2016. Ethanol extraction-based drying enhanced ESR radical detection in oranges irradiated to different ionizing radiations during storage. Postharvest Biol. Technol. 112 170-17.

Kim, B.K., Ahn, J.J., Shahbaz, H.M., Kim, C.T., Kwon, J.H., 2014. Effect of drying treatment on physical identification characteristics of irradiated seasonings. Food Anal. Methods 7 (2), 268-275.

Linke, B., Helle, N., Ammon, J., Ballin, U., Brockmann, R., Brunner, J., Delincée, H., Eisen, S., Erning, D., Eschelbach, H., Gemperlr, G., Holstein, A., Jahr, D., Kaltwasser, E., Krolls, W., Kunn, T., Kruspe, W., Meier, W., Pfordt, J., Schleich, C., Stewart, E., Vater, N., Vreden, N., Bogl, K.W., Schreiber, G.A., 1995. Elektronen-spinresonanzspektroskopische Untersuchungen zur Identifizierung bestrahlter Krustentiere und Gewurze: Durchfuhrung eines Ringversuches an Nordseekrabben, Kaisergranat und Paprikapulver. Report of the Federal Institute for Health Protection of Consumer and Veterinary Medicine. BfVV-Heft 09/1995. Bundesinstitut fur gesundheitlichen Verbaucherschutz und Veterinarmedizin, Berlin.

Linke, B., Ammon, J., Ballin, U., Brockmann, R., Brunner, J., Delincée, H., Eisen, S., Erning, D., Eschelbach, H., Estendorfer-Rinner, S., Fienitz, B., Frohmuth, G., Helle, N., Holstein, A., Jonas, K., Krolls, W., Kunn, T., Kruspe, W., Marchioni, E., Meier, W., Pfordt, J., Schleich, C., Stewart, E., Trapp, C., Vreden, N., Wiezorek, C., Bogl, K.W., Schreiber, G.A., 1996. Elektronen-spinresonanzspektroskopische Untersuchungen zur Identifizierung bestrahlter getrockneter und frischer Fruchte: Durchfuhrung eines Ringversuchs an getrockneter Feigen und Mangos sowie an frischen Erdbeeren. Report of the Federal Institute for Health Protection of Consumer and Veterinary Medicine. BfVV-Heft 03/1996 (Bundesinstitut fur gesundheitlichen Verbaucherschutz und Veterinarmedizin, Berlin.

Polovka, M., Brezova, V., Simko, P., 2007. EPR spectroscopy: a tool to characterize gamma-irradiated foods. J. Food Nutr. Res. 46 (2), 75-83.

Raffi, J., 1992. Electron Spin Resonance Intercomparison Studies on Irradiated Foodstuffs. BCR-Information. Commission of the European Communities, Report EUR/13630/en, Luxembourg.

Raffi, J., Stevenson, M.H., Kent, M., Thiery, J.M., Belliardo, J.J., 1992. European intercomparison on electron spin resonance identification of irradiated foodstuffs. Int. J. Food Sci. Technol. 27, 111-124.

Sanderson, D.C.W., Carmichael, L.A., Fisk, S., 2003a. Photostimulated luminescence detection of irradiated herbs, spices and seasonings: an international collaborative trial. AOAC Int. 5, 990-997.

Sanderson, D.C.W., Carmichael, L.A., Fisk, S., 2003b. Thermoluminescence detection of irradiated fruits and vegetables: an international collaborative trial. JAOAC Int. 86 (5), 976-982.

Sanyal, B., Ahn, J.J., Maeng, J.H., Kyung, H.K., Lim, H.K., Sharma, A., Kwon, J.H, 2014. An improved approach to identify irradiated spices using electronic nose, FTIR, and EPR spectroscopy. J. Food Sci. 79 (9), C1656-C1664.

Sanyal, B., Chatterjee, S., Variyar, P.S., Sharma, A., 2012. Application of EPR spectroscopy to identify irradiated Indian medicinal plant products. Food Sci. (N. Y.) 77 (6), C710-C718.

Schreiber, G.A., Helle, N., Schlzki, G., Linke, B., Spielberg, A., Mager, M., Bogl, K.W., 1996. In: McMurray, C.H., Stewart, E.M., Gray, R., Pearce, J. (Eds.), Interlaboratory Tests to Identify Irradiation Treatment of Various Foodstuffs via Gas Chromatographic Detection of Hydrocarbons, ESR Spectroscopy and TL Analysis. Detection Methods for Irradiated Foods- Current Status. Royal Society of Chemistry, Cambridge, pp. 98-107.

Schreiber, G.A., Helle, N., Schlzki, G., Spielberg, A., Linke, B., Wagner, U., Bogl, K.W., 1993. Intercomparisons to evaluate the suitability of gaschromatografic, electron spin resonance spectrometric and thermoluminescence methods to detect irradiated foods in routine control. Radiat. Phys. Chem. 42, 391-396.

Shahbaz, H.M., Akram, K., Ahn, J., Kwon, J., 2013. Investigation of radiation-induced free radicals and luminescence properties in fresh pomegranate fruits. J. Agric. Food Chem. 61, 4019-4025.

Yordanov, N.D., Lagunov, O., Dimov, K., 2009. EPR spectra induced by gamma-irradiation of some dry medical herbs. Radiat. Phys. Chem. 78, 277-280. 